\title{
Unacceptable status quo in access to mental health care
}

'I 'm seeing a psychiatrist." For any of us physicians who have said this (and the real number is both bigger than acknowledged and likely smaller than it should be), it immediately evokes a cloistered office where we can speak freely, at length, and regularly - with or without the aid of medications. It speaks of a relationship to say you are "seeing" someone, and that's because the relationship is important in mental health (actually, it's important in all of medicine, but we don't like to talk about it, except when it goes awry and appears in college discipline reports). For some people, expectations of "seeing a psychiatrist" may include an hour a week for months or even years, an idea that is reinforced in popular culture by cartoons, television and movies.

If this model were universally applied, with the 4000 psychiatrists in Canada seeing 40 ongoing patients per week for an hour each, there would be about 160000 people in Canada in continuing psychiatric care. The most recent data suggest that each year (and it's not the same people each year), 600000 Canadians meet criteria for a diagnosable mental disorder. Fewer than three per cent of them could be accommodated in this format of weekly treatment if that were the only model.

But of course it isn't. Psychiatrists work in hospitals and a variety of community settings as well as solo practices, providing not only ongoing care, but also consultation and indirect care. Nevertheless, my primary care colleagues regularly bemoan the lack of access to mental health services for their patients. Whether in a big city or a small town, access is a real problem, with some notable exceptions. Some family physicians I know have understandably given up on accessing psychiatric services for their patients, having depleted their personal-favourto-me accounts. So what can be done?

Arguments about simply increasing the number of psychiatrists are, in my view, stale. There will never be enough psychiatrists to address all the mental health needs of Canadians - nor should there be. Not everyone with hypertension will or should see a cardiologist. First and foremost, we should be asking not simply how many psychiatrists there are but what they do with their professional time.

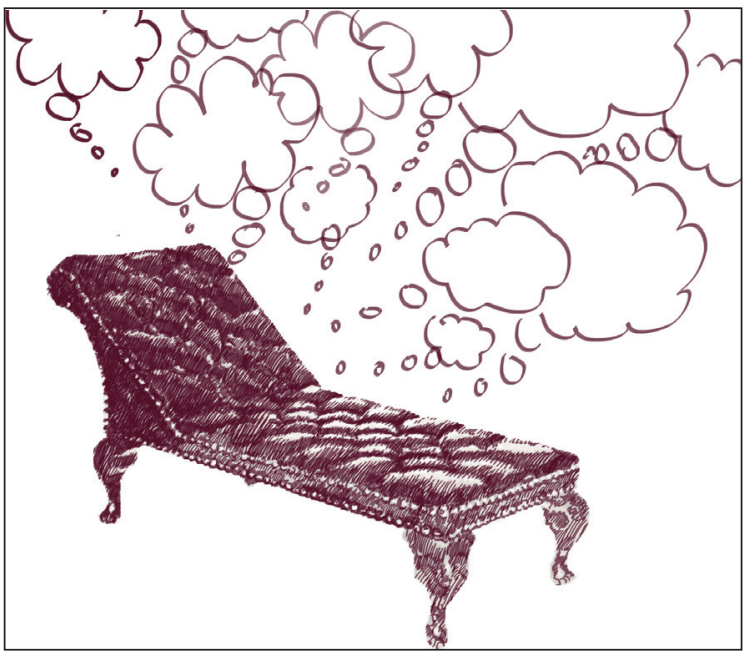

The determinants of their professional activity are multiple, including: their training, collegial and public expectation and the fee schedule. There has been, in recent years, an increased emphasis in training on short-term, evidence-based psychotherapies that allow for turnover in clinical practice, but the full impact of that has yet to be felt among practising psychiatrists. It is also yet to be incorporated into the culture of public expectation of what psychiatrists do. Finally, remuneration remains a powerful motivator of human behaviour. If there is a differential reward for seeing new patients, it will be reflected in clinical practice.

Then there is the issue of where psychiatrists do what they do. Again, while the stereotype is of a dimly lit office with an Oriental carpet, a couch and primitive carvings, the reality can be shared space in a primary care office that allows for direct and indirect care, or a televideo office that allows for consultation and follow up to isolated rural communities. Psychiatry, more than any other medical specialty, should participate actively in televideo clinical work, since we don't need to touch our patients to do our jobs and because the evidence for its palatability has been established. In Ontario, the fee schedule now provides a modest premium for televideo consultation over the usual rate - a not-so-subtle blandishment to meet a pressing clinical need.

Mental health care and psychiatry are casually used as interchangeable terms, but the reality is that the majority of mental health care (which reaches the minority of people who need it) is provided by family physicians. Are they trained, supported and remunerated in a way to optimize this?

The creation of multidisciplinary family health teams may go some way to including nonmedical professionals in the tent of publicly funded services, but whether this will translate into more accessible mental health care is still an open question. The larger question is whether we will see public funding of nonphysician mental health professionals for ongoing treatment — as has happened in Australia - while ensuring standards and accessibility.

While many Canadians benefit from good psychiatric care, the reality is that the status quo is unacceptable. As the public climate for acknowledging mental illness and its ubiquity grows, so will the expectations for timely, accessible and effective care. And so they should.

\section{David S. Goldbloom MD}

Vice-Chair

Mental Health Commission of Canada Toronto, Ont.

Have you got an opinion about this article? Post your views at cmaj.ca. Potential Salon contributors are welcome to send a query to salon@cmaj.ca

All editorial matter in CMAJ represents the opinions of the authors and not necessarily those of the Canadian Medical Association. 